\title{
Instanton contributions to the low-lying hadronic mass spectrum
}

\author{
Samuel Thomas*, Waseem Kamleh, and Derek Leinweber \\ CSSM, University of Adelaide \\ E-mail: samuel.d.thomaseadelaide.edu.au \\ waseem.kamleh@adelaide.edu.au \\ derek. leinweber@adelaide.edu.au
}

The masses of the light hadrons are calculated on in lattice QCD at various stages of stout-link smearing tuned specifically to preserve instanton-like objects. The calculation is performed using a $20^{3} \times 40$ dynamical FLIC ensemble with lattice spacing $0.126 \mathrm{fm}$ at a range of pion masses; the effect of vacuum instanton content is compared at a common pion mass of $350 \mathrm{MeV}$. We find little evidence that the instanton effect is as significant as models would suggest. In fact, the direct instanton-induced effect cannot be seen at all.

The 30th International Symposium on Lattice Field Theory

June 24 - 29, 2012

Cairns, Australia

${ }^{*}$ Speaker. 


\section{Introduction}

The instanton is well-known as a finite-action solution to the Yang-Mills equation. It is called a pseudoparticle because it is described by a location and extent parameter. Models approximating the QCD vacuum as a liquid or gas of these pseudoparticles have enjoyed some phenomenological success. A phenomenological determination of the parameters gives $\rho=0.33 \mathrm{fm}$ for the average instanton radius and $n \approx 1 \mathrm{fm}^{-4}$ for the density. Instanton effects can then explain much of the hadronic spectrum. However, it is unknown to what extent the real QCD vacuum can be approximated by an instanton model without external determination of parameters. To what extent are physical observables dependent on the topological structure?

\section{Over-Improved Smearing}

In order to examine the topological content of the QCD vacuum, we must remove the shortdistance interaction. Standard methods of doing this, by local minimisation of the gauge action, tend to unnecessarily corrode the very structures of interest due to discretisation errors.

Consider the gluonic Wilson action of a single instanton: $S=\frac{8 \pi}{g}\left\{1-\frac{1}{5}(a / \rho)^{2}+\mathscr{O}\left(a^{4}\right)\right\}$ The leading-order discretisation error in the action then induces shrinking of instantons (and similar objects) until they fall through the lattice. Even using an improved action, with larger Wilson loops having coefficients tuned to remove this leading error term; the remaining $\mathscr{O}\left(a^{4}\right)$ term is still negative and thus has the same problem. We use a modification of stout-link smearing wherein the leading order discretisation error is made positive. This is done by choosing the sum of paths $\frac{5-2 \varepsilon}{3}(1 \times 1)-\frac{1-\varepsilon}{12}(1 \times 2+2 \times 1)$ in calculating the smeared link, rather than only $(1 \times 1)$. The improvement parameter $\varepsilon$ is chosen such that $\varepsilon=1$ gives the standard action and $\varepsilon=0$ is analogous to $\mathscr{O}\left(a^{2}\right)$ improvement. A negative value of $\varepsilon$ then gives a positive $\mathscr{O}(a)$ leading error term and preserves topological structure. We take $\varepsilon=-0.25$ and an isotropic smearing parameter $\rho_{s m}=0.06$ as in [2]. This value of $\varepsilon$ is small enough that instantons are also not induced to grow unphysically large under smearing. This combination of parameters gives a dislocation threshold of 1.97a. Once instanton-like objects with a radius under this threshold are removed, the only nontrivial change in the vacuum comes from pairwise annihilation of instanton-anti-instanton pairs.

\section{Simulation Details}

The lattices used in this study are $20^{3} \times 40$ 2-flavour dynamical FLIC, using the partiallyquenched approximation for the valence quarks. The lattice coupling is $\beta=3.94$ and sea-quark $\kappa=0.1324$; these values provide dynamical masses similar to the strange quark mass. The spacing, $a=0.126$ is set on the unsmeared configurations using the string tension. This corresponds to a lattice size of $2.52^{3} \times 5.04 \mathrm{fm}$. We use this same value at all levels of smearing as the string tension is a fundamentally non-local property. Quark propagators are calculated using the FLIC action [4] with boundary conditions periodic in space and fixed in the time direction to disallow hadron propagation past the temporal limit of the lattice. The fermionic source was inserted at $t=10$ to be far enough from this fixed boundary to ensure that edge conditions are minimal. Fifty sweeps of Gaussian smearing are used in the source. 


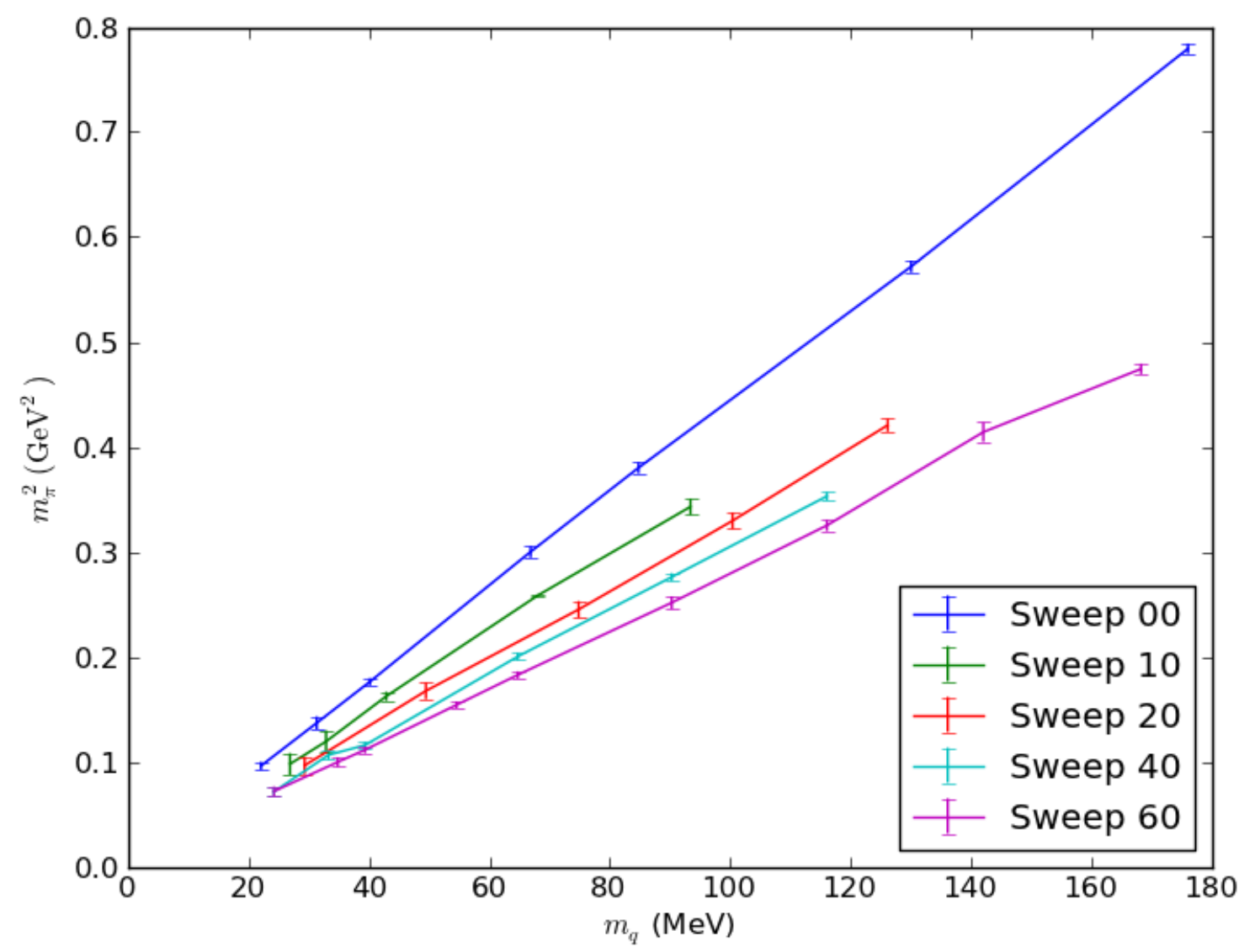

Figure 1: The Gell-Mann-Oakes-Renner relation on the smeared fields

The Gell-Mann-Oakes-Renner relation [1], indicates $m_{\pi}^{2}=2\langle q \bar{q}\rangle m_{q} / f_{\pi}^{2}$ (we use $m_{u}=m_{d}=m_{q}$ in our propagators). The quark mass is defined by $m_{q}=\frac{1}{2 a}\left\{\frac{1}{\kappa}-\frac{1}{\kappa_{c r}}\right\}$, where the critical value is determined by extrapolation to vanishing pion mass. The value of $\kappa_{c r}$ rapidly approaches its treelevel value of $1 / 8$ under smearing;

We see from Fig. 1 that the Gell-Mann-Oakes-Renner relation continues to hold on the smeared configurations. We will thus use the squared pion mass as a physically relevant indicator of the quark mass. For some alternative transformations of the gauge field, for example, vortex-removed configurations [3], the pion loses its Nambu-Golstone nature.

The change in the slope of $m_{\pi}^{2}$ vs. $m_{q}$ in Fig. 1 is indicative of a decrease in the quark condensate under smearing (although the pion decay constant is also expected to change under smearing, so a quantitative treatment is nontrivial). The quark condensate is proportional to the instanton number in an instanton model; this is an indication of the rate of pairwise pseudoparticle annihilation.

The rho, nucleon, and Delta baryon masses were also calculated at a range of pion masses. All were seen to retain the form of their quark-mass dependence on the smeared configurations; with the exception that smearing allowed calculation at lower quark masses than otherwise feasible. Also there were some indications that the Delta became unbound at sufficiently small pion 


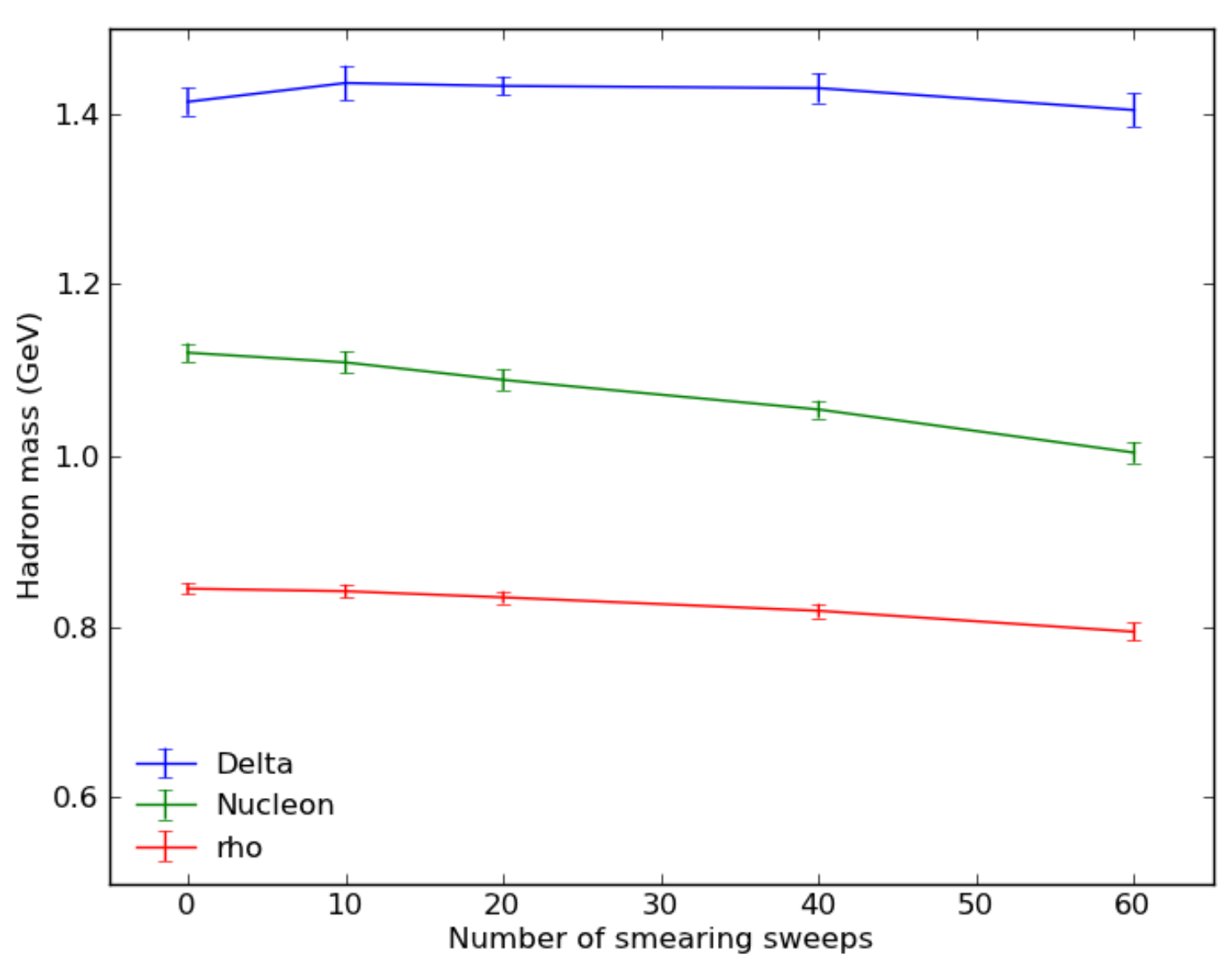

Figure 2: Smearing dependence of hadron masses at a common squared pion mass of $0.150 \mathrm{GeV}^{2}$

masses. In order to analyse the smearing-dependence, the bare quark mass was tuned to reproduce a common squared pion mass of $0.150 \mathrm{GeV}^{2}$. In order to isolate a clear signal for the Delta, it proved necessary to use a different fermionic source on the unsmeared configurations compared to all others. On the smeared configurations the same 50-sweep Gaussian smeared source used for the other hadrons was sufficient, but on the unsmeared configurations a larger source (greater than 100 sweeps) had better overlap with the ground state. This is an indication that the size of the Delta changes more under smearing than the other hadrons.

\section{Conclusions}

Dynamical mass generation is lost by the removal of very small objects; this happens mainly within the first 10 sweeps. Between 10 and 60 sweeps, the main change in the vacuum comes from instanton-anti-instanton pair annihilation. An instanton-based model of the vacuum implies an attractive contribution from such a pair in the pion and nucleon channels, and no lowest-order interaction with either the Delta or the rho [5]. Instanton-like objects such as those we see under smearing, while not exact Dirac zero-modes, are strongly correlated to low-lying modes [6]; the same theory then applies. Thus, such a model would predict an increase in the nucleon mass under 
smearing as this attraction is lost (this was seen in [7], but that study used a cooling procedure which destroys topological structure). The result we see in Fig. 2 is that, while both the nucleon and Delta masses decrease under smearing (as dynamical mass generation is lost), the nucleon in fact decreases faster - the decreasing instanton interaction is not seen. Hence, we can conclude that the instanton-like effects observed in the QCD vacuum revealed by smearing are different from the model expectations.

In fact, the results seem to be more easily explained by a simple constituent-quark model with one-gluon-exchange hyperfine splitting inversely proportional to the product of the quark masses. As the constituent quark mass is decreased by the change in the quark condensate, this hyperfine splitting between the Delta and the nucleon is increased, resulting in behaviour similar to that seen in Fig. 2. The change we find in this constituent quark mass is consistent with the change in the overlap quark propagator under similar smearing [8].

\section{Acknowledgments}

This research was undertaken on the NCI nation facility in Canberra, Australia, which is supported by the Australian Commonwealth Government. We also acknowledge eResearch SA for generous grants of supercomputing time. This research is supported by the Australian Research Council.

\section{References}

[1] M. Gell-Mann, R. J. Oakes, and B. Renner, Phys. Rev. 175, 2195 (1968).

[2] P. J. Moran and D. B. Leinweber, Phys. Rev. D 77, 094501 (2008), [hep-lat/ 0801.1165$]$

[3] E.-A. O'Malley, W. Kamleh, and D. B. Leinweber, [hep-lat/1112.2490]

[4] S. O. Bilson-Thompson, D. B. Leinweber, and A. G. Williams, Ann. Phys. 304, 1 (2003), [hep-lat/0203008]

[5] T. Schäefer and E. V. Shuryak, Rev. Mod. Phys. 70, 2 (1998).

[6] D.-J. Kusterer, J. Hedditch, W. Kamleh, D. B. Leinweber, and A. G. Williams, Nucl. Phys. B628, 2002, [hep-lat/0111029]

[7] M. Chu, J. Grandy, S. Huang, and J. W. Negele, Phys. Rev. D49, 6039 (1994), [hep-lat/9312071]

[8] D. Trewartha, Master's Thesis, University of Adelaide, 2012, and these proceedings. 